Check for updates

Cite this: RSC Adv., 2019, 9, 9620

Received 10th January 2019

Accepted 11th February 2019

DOI: $10.1039 / c 9 r a 00230 h$

rsc.li/rsc-advances

\section{Three-dimensional $\mathrm{N}$-doped graphene aerogel- supported Pd nanoparticles as efficient catalysts for solvent-free oxidation of benzyl alcohol}

\begin{abstract}
Qiuli Wei, Tongjun Liu, Yuanyuan Wang (iD * and Liyi Dai
Herein, three-dimensional (3D) nitrogen-doped graphene with large surface areas and abundant porous structures was prepared by a hydrothermal synthesis method, which served as a novel support to enhance the catalytic properties of noble metal catalysts for the solvent-free selective oxidation of benzyl alcohol. The as-prepared samples were characterized by X-ray diffraction (XRD), scanning electron microscopy (SEM), transmission electron microscopy (TEM), X-ray photoelectron spectroscopy (XPS), Raman spectroscopy, Fourier transform infrared (FTIR) spectroscopy, and Brunauer-Emmett-Teller (BET) method. The results clearly showed that the introduced $\mathrm{N}$-containing group prevented the aggregation of graphene sheets and provided more structural defects to maximize the number of exposed active sites. The threedimensional structure can provide a unique porous structure and large specific surface area. Moreover, the three-dimensional structure makes the recycling and reuse of the catalyst easier. The combination of these properties results in the reduction of the average particle size of metal palladium to $3.2 \mathrm{~nm}$; this significantly increases the catalytic activity of the catalyst. The three-dimensional $\mathrm{N}$-doped graphene aerogel-supported Pd nanoparticle (3D Pd/NRGO) composites exhibit excellent catalytic activity for the solvent-free selective oxidation of benzyl alcohol to benzaldehyde by molecular oxygen at $90{ }^{\circ} \mathrm{C}$ for 3 hours under atmospheric pressure, resulting in a $72.2 \%$ conversion of benzyl alcohol with $94.5 \%$ selectivity for benzaldehyde. In addition, the catalytic efficiency shows no obvious loss even after six repeated cycles. Thus, 3D Pd/NRGO can be used as an efficient, easily separable, recyclable, and stable catalyst for the solvent-free selective oxidation of benzyl alcohol under relatively mild conditions.
\end{abstract}

\section{Introduction}

Metal nanocatalysts, especially noble metal (Ag, Pt, Pd, $\mathrm{Au}, \mathrm{Ru}$, etc.) nanocatalysts, dispersed on supports play an important role in many fields. ${ }^{\mathbf{1 - 4}}$ Moreover, nano-sized metal nanoparticles may be the real active phase..$^{5-7}$ However, the applications of nanometer-sized metal particles are significantly hampered by their inhomogeneous particle sizes, poor cycling performances and aggregation, ${ }^{8}$ leading to low stability and catalytic activity. To overcome the abovementioned problems, one efficient strategy is to immobilize these metal nanoparticles (NPs) on an appropriate catalyst carrier. ${ }^{9}$ Studies have shown that the performance of metal catalysts depends to a large extent on their interaction with the support. ${ }^{10}$

Graphene as a two-dimensional single-layer $\mathrm{sp}^{2}$-bonded carbon material has received significant attention because of its potential application in the field of catalysis ${ }^{\mathbf{1 1 2} \mathbf{1 2}}$ due to its properties such as large specific surface area, thermal characteristics and outstanding electrical conductivity; ${ }^{\mathbf{1 2}}$ however, its

College of Chemistry and Molecular Engineering, East China Normal University, 500 Dongchuan Road, Shanghai 200241, China. E-mail: ecnu_yywang@163.com; Fax: +86-021-54340133; Tel: +86-021-54340133 perfect binary symmetry structure results in the lack of modulation of its properties; this limits its performance of anchoring the catalyst nanoparticles. ${ }^{\mathbf{1 1 , 1 3 , 1 4}}$ More importantly, the interaction between the metal catalyst and the intrinsic graphene is not strong, which is not conducive to improving the catalytic performance of the metal catalyst. ${ }^{15}$ In general, an efficient way is to break the symmetrical structure of graphene by introducing a heteroatom, which can significantly change the electron distribution and local bonding environment on the surface of graphene, leading to increased loading sites for anchoring the catalyst nanoparticles on the graphene lattice. ${ }^{16}$ Displacement doping involves the replacement of the carbon atoms in the graphene honeycomb lattice by atoms, such as nitrogen, ${ }^{\mathbf{1 7}}$ boron, ${ }^{18}$ phosphorus ${ }^{19,20}$ and sulfur, ${ }^{21}$ with different valence electron numbers. Nitrogen doping of graphene has been intensively investigated in the last few years. ${ }^{22,23} \mathrm{~N}$, next to $\mathrm{C}$ in the periodic table, is similar in size to the carbon atom, which can be easily embedded in the graphene lattice to achieve doping; ${ }^{24}$ moreover, $\mathrm{N}$ has higher electronegativity (3.04) relative to $\mathrm{C}$ (2.55). Therefore, the introduction of $\mathrm{N}$ into graphene can modify the local electronic structure and induce polarization in the carbon network. ${ }^{25}$ When graphene is doped with $\mathrm{N}$ atoms, the soliton pair of $\mathrm{N}$ atoms can detach the $\mathrm{sp}^{2}$ - 
hybridized $\mathrm{C}$ atoms from their conjugated systems, activate the $\pi$ electrons, and achieve charge distribution and surface defect degree on the surface of the graphene carbon material. ${ }^{26}$ In other words, effective modulation helps to change the spin density and charge distribution of adjacent carbon atoms. On the one hand, $\mathrm{N}$ doping can provide active sites for the adsorption and effective reduction of $\mathrm{O}_{2}$ molecules; on the other hand, it also affects the nucleation and growth of graphenesupported metallic nanoparticles and reduces the particle size. Via its lone pair of electrons, $\mathrm{N}$ can form a coordination bond with metal atoms; this effectively strengthens the binding force between the carrier and the metallic nanoparticles such that the dispersibility of the nanoparticles is greatly improved and the activity and stability of the catalyst are significantly upgraded. Thus, nitrogen-doped graphene-loaded Pd nanoparticles have been used as catalysts in some reactions. ${ }^{27-29}$ The excellent catalytic activity of these catalysts is attributed to the increased defect level, which leads to a reasonable modification of electron trajectories, and well-dispersed Pd nanoparticles on the $\mathrm{N}$-doped graphene support, which lead to high active surface areas and thereby higher catalytic activities.

In addition to changing the composition of the catalyst as abovementioned, increasing the specific surface area of the catalyst and improving the mass transfer in the catalytic process by constructing a reasonable porous structure are effective methods for improving the catalytic activity of a graphene-based catalyst. Therefore, the self-assembly of two-dimensional (2D) graphene sheets into three-dimensional (3D) structures has received extensive attention. The $3 \mathrm{D}$ structures provide graphene materials with high specific surface areas; moreover, due to the combination of the $3 \mathrm{D}$ porous structures and the excellent intrinsic properties of graphene, the 3D porous interconnected network structure facilitates the provision of a special reactive microenvironment and conductive multi-path for fast mass and electron transport kinetics. This further illustrates that the three-dimensional $\mathrm{N}$-doped graphene is a good catalytic carrier for improving the catalytic activity. However, to date, most of the studies on $\mathrm{N}$-doped reduced graphene oxide have been focused on fuel cells ${ }^{15,26,32}$ and electrochemical $^{13,16,24,30,31}$ applications; moreover, although these materials may have good catalytic activities, they are difficult to be recycled and reused. Therefore, studies on the use of threedimensional $\mathrm{N}$-doped graphene-loaded metal nanoparticle composites as catalysts have not been reported; thus, it is attractive to explore a simple method for the fabrication of three-dimensional $\mathrm{N}$-doped graphene-supported metal nanoparticle composites as catalysts, which can uniformly distribute small-sized metal nanoparticles on the surface of NRGO sheets.

In this study, a nitrogen-doped graphene metal catalyst (3D Pd/NRGO) with a special three-dimensional network structure was prepared by a hydrothermal synthesis $\operatorname{method}^{33}$ using $N$ methylpyrrolidone (NMP) as a nitrogen source. ${ }^{31,34}$ This catalyst has been applied in the selective oxidation reactions of benzyl alcohol in the liquid phase via an ideal green process using molecular oxygen as an oxidant; moreover, no solvent or additives have been used in this reaction, which is an important alcohol oxidation reaction in industries due to the demand for benzaldehyde that is used as an intermediate in the production of fine chemicals, fragrances and flavouring additives. ${ }^{35-38}$ This material has not only the advantages of two-dimensional graphene materials, but also other unique characteristics such as loose porosity, low density, large surface-to-volume ratio, strong mechanical properties and good electrical conductivity; moreover, NMP is similar to a stent, further extending the distance between graphene sheets such that the three-dimensional network structure has a larger surface area. The large specific surface area and more active sites due to $\mathrm{N}$ doping can effectively avoid the agglomeration of palladium nanoparticles, improve the dispersion of the nanoparticles, and thereby synchronously provide high selectivity and conversion. Moreover, this catalyst can be conveniently recycled.

\section{Experimental}

\section{Materials}

Natural graphite flakes (1200 mesh) were obtained from Qingdao Jinrilai Graphite Co., Ltd. Concentrated $\mathrm{H}_{2} \mathrm{SO}_{4}$ (98\%), $\mathrm{KMnO}_{4}, \mathrm{P}_{2} \mathrm{O}_{5}, \mathrm{H}_{2} \mathrm{O}_{2}(30 \%)$ solution, $\mathrm{K}_{2} \mathrm{~S}_{2} \mathrm{O}_{8}, \mathrm{PdCl}_{2}$, and $\mathrm{NaBH}_{4}$ were purchased from Aladdin Chemical Reagent Co., Ltd. and used as received. L-Ascorbic acid was supplied from Sinopharm Chemical Reagent Co. Other organic substrates were supplied from Sinopharm Chemical Reagent Co., Ltd. and Aladdin Reagent Co., Ltd. The rest of the materials were obtained from local suppliers. All chemicals are used without further purification. Deionized water was used throughout the experiments.

\section{Preparation of graphene oxide (GO)}

Graphene oxide was prepared by a modified Hummers method using a 1200-mesh natural graphite powder. ${ }^{39}$ Briefly, graphite powder $(5 \mathrm{~g}), \mathrm{K}_{2} \mathrm{~S}_{2} \mathrm{O}_{8}(2.5 \mathrm{~g})$ and $\mathrm{P}_{2} \mathrm{O}_{5}(2.5 \mathrm{~g})$ were added to concentrated sulfuric acid $(100 \mathrm{~mL})$ followed by stirring at $80{ }^{\circ} \mathrm{C}$ for about $6 \mathrm{~h}$. After being cooled down to room temperature, the mixture was slowly added to deionized water (about $1 \mathrm{~L}$ ) until the $\mathrm{pH}$ value was close to seven, and the solid was obtained by vacuum filtration. Then, the solid was washed three times with deionized water. After drying the solid overnight at $50{ }^{\circ} \mathrm{C}$, preoxidized graphite compounds were obtained. After this, the preoxidized expanded graphite compounds were added to $120 \mathrm{~mL}$ concentrated sulfuric acid, and then, $15 \mathrm{~g} \mathrm{KMnO}_{4}$ was slowly added under continuous stirring in an ice bath. Then, the ice bath was removed, and the mixture was stirred at $35{ }^{\circ} \mathrm{C}$ for about $2 \mathrm{~h}$. After this, $1000 \mathrm{~mL}$ of deionized water was slowly added. After the addition of $230 \mathrm{~mL}$ water, the mixture was further stirred for $15 \mathrm{~min}$ while increasing the temperature to $98{ }^{\circ} \mathrm{C}$. Finally, the remaining deionized water was added followed by a slow addition of $12.5 \mathrm{~mL}$ of $\mathrm{H}_{2} \mathrm{O}_{2}$ (30\%). With the addition of hydrogen peroxide, the mixture changed from dark brown to yellow. After the process of oxidation, the mixture was repeatedly washed with an $\mathrm{HCl}$ solution and water $(1: 10)$ by repeated centrifugation. ${ }^{8}$ Then, the product was purified by dialysis for one week using a dialysis membrane with a molecular weight cut off of 8000-10 $000 \mathrm{~g} \mathrm{~mol}^{-1}$; this removed the remaining acid and the residual salts. To obtained smaller 
sheets, graphite oxide was exfoliated by an ultrasonic cell crusher.

\section{Preparation of the catalyst}

To investigate the influence of the quantities of $N$-methylpyrrolidone (NMP) on the catalytic effect, catalysts with different NMP/GO mass ratios were prepared: $0: 1$ (which means the mass ratio of NMP to GO was $0: 1$, catalyst-0), $0.8: 1$ (catalyst-1), $1.2: 1$ (catalyst-2), $1.5: 1$ (catalyst-3), $1.8: 1$ (catalyst-4), and $2.2: 1$ (catalyst-5). Herein, the preparation of catalyst-3 was taken as an example. Typically, $20 \mathrm{mg}$ of GO was dispersed in $10 \mathrm{~mL}$ of deionized water. To exfoliate the GO nanosheets, the solution was sonicated for 30 minutes. Then, $300 \mu \mathrm{L}$ NMP solution (including NMP $30 \mathrm{mg}$ ) was added to the abovementioned aqueous solution followed by ultrasonication for 20 minutes, and the mixture was stirred for ten minutes to transform the turbid mixture into a homogeneous and transparent solution. ${ }^{40}$ Subsequently, $2.67 \mathrm{mg} \mathrm{PdCl}_{2}$ was added followed by ultrasonication for another $30 \mathrm{~min}$. The mixture was stirred overnight at room temperature. Then, $60.2 \mu \mathrm{L} 0.5$ molL ${ }^{-1} \mathrm{NaBH}_{4}$ solution was added to the abovementioned solution, and the solution changed from brown to black. After stirring for $1 \mathrm{~h}, 0.16 \mathrm{~g}$ of $\mathrm{L}$-ascorbic acid was added followed by stirring at room temperature for $20 \mathrm{~min}$. The mixture was then maintained at $90{ }^{\circ} \mathrm{C}$ for $1 \mathrm{~h}$ and then cooled down to room temperature. Finally, the resulting catalyst-3 hydrogel was washed several times with ethanol and deionized water and then lyophilized to an aerogel for further use. The preparation steps for other catalysts are similar, except that the mass ratio of NMP to GO is changed.

\section{Catalyst characterization}

X-ray diffraction (XRD) patterns were obtained using the Bruker AXS D8 diffractometer at the scanning speed of $15^{\circ} \mathrm{min}^{-1}$. Raman spectroscopy was conducted using Thermo DXR with a $532 \mathrm{~nm}$ laser excitation. X-ray photoelectron spectroscopy (XPS) results were obtained using the PHI-5500 spectrometer with $\mathrm{Al} \mathrm{K} \alpha \mathrm{X}$-ray radiation as the X-ray excitation source. Transmission electron microscopy (TEM) was conducted using JEM-2100F, and scanning electron microscopy (SEM) was performed using JSM-5610LV. Inductively coupled plasma atomic emission spectroscopy (ICP-AES) measurements were conducted using the Thermo Scientific iCAP 6300 instrument. Infrared spectra results were obtained using the NEXUS 670 Fourier transform infrared (FTIR) spectrometer. The $\mathrm{N}_{2}$ adsorption-desorption isotherms were acquired using the Micromeritics Tristar 3000 analyzer. The specific surface areas and pore size distributions of the catalysts were calculated by the Brunauer-Emmett-Teller (BET) and Barrett-Joyner-Halenda (BJH) methods, respectively. Elemental compositions were obtained via the CHN elemental analysis (Elementar Vario EL III).

\section{Catalytic activity measurements}

The catalytic performance for the selective oxidation of benzyl alcohol to benzaldehyde was tested under normal pressure. The liquid-phase oxidation of benzyl alcohol was carried out in a magnetically stirred three-necked flask $(25 \mathrm{~mL})$ equipped with a reflux condenser and an oil bath using molecular oxygen as the oxidant; the oil bath was heated in advance to a suitable temperature. The magnetic stirring rate was 1200 rpm. Typically, $48.15 \mathrm{mmol}$ of benzyl alcohol and Pd/NRGO catalyst were added to the reactor, and oxygen was continuously blown under atmospheric pressure. The substrate to metal molar ratio was about 5100, and the flow rate of oxygen was $20 \mathrm{~mL} \mathrm{~min}^{-1}$. After the reaction, when the temperature of the mixture was near room temperature, the catalyst was taken out from the mixed solution, thoroughly washed with ethanol and deionized water, and then freeze-dried for the next run. The reaction product was subjected to the same analysis by gas chromatography (GC2014, shimadzu).

\section{Results and discussion}

\section{Catalytic performance of the catalysts}

The catalytic activity of the six catalysts with different NMP/GO mass ratios towards the oxidation of benzyl alcohol was evaluated, and the results were compared, as shown in Fig. 1a. As illustrated, the conversion and selectivity of the catalyst- 0 were $42.9 \%$ and $84.9 \%$, respectively, and the yield was only $36.4 \%$. As the mass ratio of NMP to GO was increased, the yield of the catalyst also increased from $36.4 \%$ to $68.2 \%$. The optimum catalyst was catalyst-3 (with the NMP/RGO mass ratio of $1.5: 1$ ); the benzyl alcohol conversion was high at about $72.2 \%$, and the benzaldehyde selectivity was about $94.5 \%$. After this, when the mass ratio of NMP to GO was continuously increased, the yield of the catalyst did not significantly change. This indicated that the 3D Pd/NRGO catalysts showed higher catalytic activity than the $3 \mathrm{D} \mathrm{Pd} / \mathrm{RGO}$ catalysts. This means that the attachment of NMP can effectively improve the reaction activity for the benzyl alcohol oxidation reaction. This high catalytic activity may be due to the polarization distribution of spin and charge density caused by the introduction of $\mathrm{N}$ heteroatoms. ${ }^{\mathbf{4 1 , 4 2}}$

Fig. 1b summarizes the influence of the Pd content on the benzyl alcohol oxidation catalyzed by Pd/NRGO. Under the same reaction conditions, when the theoretical loading amount of Pd on the catalyst- 3 was increased from $4 \mathrm{wt} \%$ to $8 \mathrm{wt} \%(0.8-$

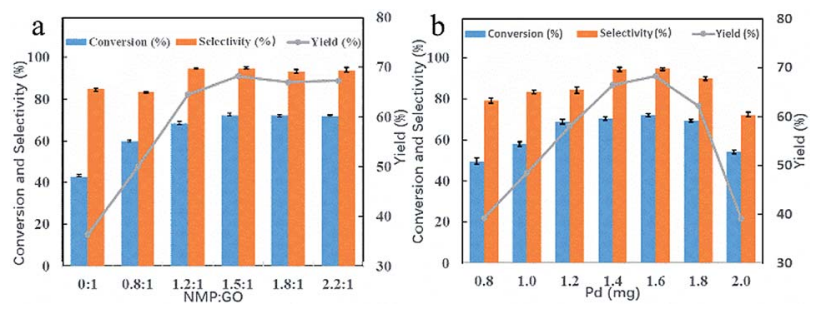

Fig. 1 (a) The catalytic performance of the six catalysts with different NMP/GO mass ratios and (b) the catalytic performance of catalyst- 3 with different $\mathrm{Pd}$ contents. Reaction conditions: temperature, $90^{\circ} \mathrm{C}$;

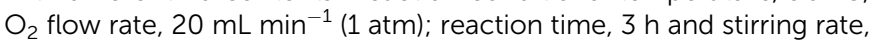
$1200 \mathrm{rpm}$. Yield $=$ the product of benzyl alcohol conversion and benzaldehyde selectivity. 
$1.6 \mathrm{mg}$ ), the yield of the benzaldehyde product increased from $39.1 \%$ to $68.2 \%$. However, a further increase in the loading of $\mathrm{Pd}$ on the catalyst did not increase the yield. This may be due to the aggregation of excess palladium nanoparticles that reduces the catalytic activity of the precious metal and increases the cost of the reaction. Therefore, for catalyst-3, the optimal loading is $8 \mathrm{wt} \%$ of the theoretical loading, which is basically the same as the actual loading of $7.8 \mathrm{wt} \%$ measured by ICP, and the catalyst3 shows a maximal yield of $68.2 \%$.

The catalytic activity of catalyst- 3 at different temperatures is shown in Fig. 2 a. At $70{ }^{\circ} \mathrm{C}$, the yield was only $18.2 \%$. When the temperature was increased from $70{ }^{\circ} \mathrm{C}$ to $90{ }^{\circ} \mathrm{C}$, the yield of benzaldehyde almost linearly increased. When the temperature was increased to $90{ }^{\circ} \mathrm{C}$, the yield of benzyl alcohol significantly increased to $68.2 \%$. The conversion and selectivity were around $72.2 \%$ and $94.5 \%$, respectively. When the temperature was gradually increased from $90{ }^{\circ} \mathrm{C}$ to $120{ }^{\circ} \mathrm{C}$, the conversion of benzyl alcohol also increased, whereas the selectivity of benzaldehyde decreased; this resulted in a decrease in the yield; one reason for this phenomenon might be that as the temperature increased, some functional groups of graphene oxide were decomposed;3-46 this resulted in the aggregation of palladium metal particles that reduced the selectivity of benzaldehyde. Therefore, $90{ }^{\circ} \mathrm{C}$ was chosen as the optimum reaction temperature for the catalytic reaction of the catalyst.

Fig. 2b shows the catalytic performance of the catalyst-3 at different reaction times. It was observed that during the first three hours of the reaction, the conversion of benzyl alcohol increased from $8.6 \%$ to $72.2 \%$ with an increase in reaction time, and the yield increased from $8.5 \%$ to $68.2 \%$. However, as the reaction time continued to increase, the selectivity of benzaldehyde decreased from $94.5 \%$ for the reaction time of $3 \mathrm{~h}$ to $88.6 \%$ for the reaction time of $4 \mathrm{~h}$. The yields at the reaction times of $3 \mathrm{~h}$ and $4 \mathrm{~h}$ were $68.2 \%$ and $68.5 \%$, respectively, and there was only a slight increase in the yields. Therefore, from the perspective of energy conservation, the reaction time was set at $3 \mathrm{~h}$.

\section{Catalyst characterization}

The structural differences in the defects and functional groups of the 3D Pd/RGO and 3D Pd/NRGO samples were characterized by Raman spectroscopy, as shown in Fig. 3a. In these spectra, the intensity of the $\mathrm{D}$ band located at about $1345 \mathrm{~cm}^{-1}$

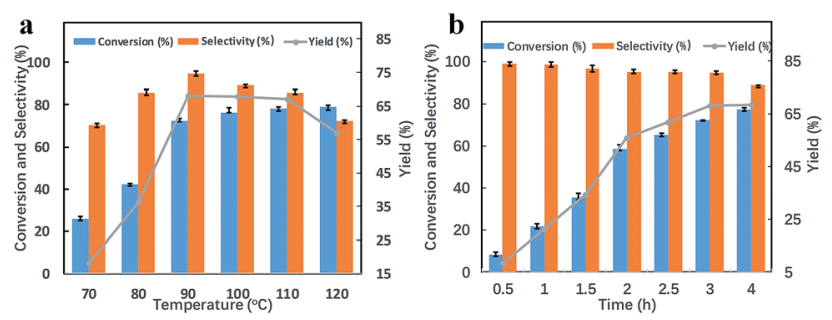

Fig. 2 (a) The catalytic performance of catalyst-3 at different temperatures and (b) different reaction times. Reaction conditions: $\mathrm{O}_{2}$ flow rate, $20 \mathrm{~mL} \mathrm{~min}^{-1}$ (1 atm); and stirring rate, $1200 \mathrm{rpm}$. Yield = the product of benzyl alcohol conversion and benzaldehyde selectivity. directly correlates with the amount of $\mathrm{sp}^{3}$ bonding present in graphene as well as the defects in the $\mathrm{sp}^{2}$ carbons caused by the lattice motion of the center of the Brillouin zone that is usually used to indicate defects in a graphene material sample. Moreover, the intensity of the $\mathrm{G}$ band at $1575 \mathrm{~cm}^{-1}$ correlates with the degree of the $\mathrm{E}_{2 \mathrm{~g}}$ vibration mode present in the $\mathrm{sp}^{2}$ hybridized carbon atoms within the hexagonal framework of graphene, and this peak is typically strongly affected (reduced) by defects, heterogeneous doping, or multilayer stacking of the graphene sheets. ${ }^{47-49}$ It is easy to accept the peak position and peak width are used to analyze the doping level. Generally, the relative intensity ratio of the $\mathrm{D}$ band $\left(I_{\mathrm{D}}\right)$ to the $\mathrm{G}$ band $\left(I_{\mathrm{G}}\right)$ reflects the degree of graphitization and the size of any structural defects or graphitic clusters existing in disordered carbon. From the Raman spectra, the values of the $I_{\mathrm{D}} / I_{\mathrm{G}}$ ratio (Table 1) were calculated to be $1.42,1.42,1.45,1.47,1.49$, and 1.51 for the catalyst- 0 , catalyst- 1 , catalyst- 2 , catalyst- 3 , catalyst4 , and catalyst-5, respectively. In other words, the $I_{\mathrm{D}} / I_{\mathrm{G}}$ ratio increases after $\mathrm{N}$ doping; this indicates that the doping of $\mathrm{N}$ can increase the defects in graphene. Moreover, this increase in the defect sites will accelerate the deposition of palladium nanoparticles. In addition, the positions of the $\mathrm{G}$ peak in the Raman spectra of the samples A (catalyst-0), B (catalyst-1), C (catalyst-2), D (catalyst-3), E (catalyst-4) and F (catalyst-5) are $1570 \mathrm{~cm}^{-1}, 1573 \mathrm{~cm}^{-1}, 1575 \mathrm{~cm}^{-1}, 1579 \mathrm{~cm}^{-1}, 1580 \mathrm{~cm}^{-1}$ and $1583 \mathrm{~cm}^{-1}$, respectively. The shift in the peak position is related to the type of doping: n-type doping, such as quaternary ammonium salt-type $\mathrm{N}$ doping, causes the $\mathrm{G}$ peak to move to lower wavenumber range, whereas p-type doping, such as pyridine-type and pyrrole-type $\mathrm{N}$ doping, causes the $\mathrm{G}$ peak to move to high wavenumber range; moreover, it can be seen in Fig. 3a that p-type doping is dominant in the nitrogen-doped graphene hydrogel prepared by the hydrothermal method, and pyridinic and pyrrolic $\mathrm{N}$ atoms are obtained; ${ }^{18}$ however, this finding needs to be confirmed using the data obtained via the XPS test.

The crystalline phase of the catalyst was characterized by powder XRD. Fig. 3b exhibits the XRD patterns of the Pd/RGO and Pd/NRGO catalysts. As shown, a broad peak-shaped signal appears between $20^{\circ}$ and $30^{\circ}$, which corresponds to the $\left(\begin{array}{lll}0 & 0 & 2\end{array}\right)$ diffraction peak of $\mathrm{C.}^{50}$ There is no significant change in this peak before and after doping. As is known, the doping of $\mathrm{N}$ does not destroy the hexagonal planar structure of graphitic carbon. The diffraction peaks at $40^{\circ}, 46^{\circ}$ and $68^{\circ}$ are attributed to the $(1$

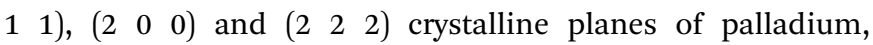

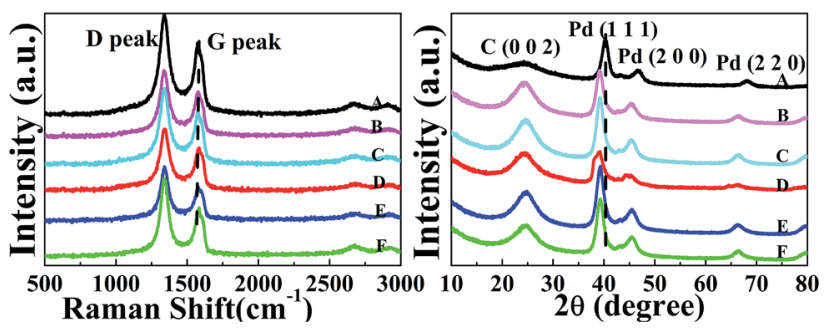

Fig. 3 (a) Raman spectra and (b) XRD patterns: catalyst-O (A), catalyst1 (B), catalyst-2 (C), catalyst-3 (D), catalyst-4 (E), and catalyst-5 (F). 
respectively (JCPDS card no. 05-0681). Furthermore, with an increase in the $\mathrm{N}$ doping amount, the characteristic diffraction peaks of the Pd ( $\left.\begin{array}{lll}1 & 1 & 1\end{array}\right)$ crystal plane in the 3D Pd/NRGO catalyst experience an obvious shift towards smaller angle as compared to the case of the $3 \mathrm{D} \mathrm{Pd} / \mathrm{RGO}$ (catalyst- 0 (A)). This indicates that the particle size of $\mathrm{Pd}$ in $3 \mathrm{D} \mathrm{Pd} / \mathrm{NRGO}$ is smaller when compared with that in the $3 \mathrm{D} \mathrm{Pd} / \mathrm{RGO}$ composite. This means that the distance between Pd atoms increases as the particle size decreases. ${ }^{8,51}$ By fitting the Pd ( $\left.\begin{array}{lll}1 & 1 & 1\end{array}\right)$ peak via the Scherrer equation, the average size of the Pd nanoparticles supported on the undoped (catalyst- 0 (A)) and N-doped graphene (catalyst-3 (D)) composites was calculated to be about $7.5 \mathrm{~nm}$ and $3.2 \mathrm{~nm}$, respectively. These results are consistent with those of the TEM statistical particle size analysis.

Controlling the size and dispersion of the Pd NPs on graphene is very meaningful for the application of these NPs in benzyl alcohol oxidation. Fig. 4 represents the TEM images and the particle-size distribution of the catalyst-0 (a), catalyst-1 (b), catalyst-2 (c), catalyst-3 (d), catalyst-4 (e), and catalyst-5 (f). Fig. 4a shows the TEM image of the catalyst that is not doped with $\mathrm{N}$, whereas Fig. $4 \mathrm{~b}-\mathrm{f}$ show the TEM images obtained after $\mathrm{N}$ doping. As shown in these TEM images, the dispersion of the Pd NPs in the 3D Pd/RGO (catalyst-0 (a)) catalyst was extremely non-uniform, and obvious agglomeration occurred. The average particle size was $7.13 \mathrm{~nm}$. From Fig. 4a, it is clear that the particle size of Pd nanoparticles is smaller in 3D Pd/NRGO; in addition, most of the Pd nanoparticles are uniformly dispersed on the NRGO surface; this is advantageous for obtaining a large number of surface active regions. Based on the statistics of the particle size analysis, the average particle diameters, as obtained from Fig. 4b, c, d, e, and f, are $5.48 \mathrm{~nm}, 4.86 \mathrm{~nm}, 3.21 \mathrm{~nm}$, $3.87 \mathrm{~nm}$, and $4.34 \mathrm{~nm}$, respectively. A strong interaction can be observed between nitrogen heteroatoms and palladium in $\mathrm{N}$ doped graphene, which can enhance the controlled nucleation of palladium, promote a higher dispersion of Pd NPs, and reduce the particle size of the metal nanoparticles, thereby increasing the catalytic activity. ${ }^{30}$ Moreover, when the mass ratio of NMP to GO reaches 1.5, the particle size of the Pd NPs is smallest, and the catalytic efficiency for the benzyl alcohol oxidation is highest. However, after the mass ratio of NMP to GO reaches up to 1.5, the particle size of the Pd NPs does not continue to decrease. Based on the TEM images and catalytic effect, it could be speculated that the excessive NMP attached to the graphene sheets might recombine through intermolecular interactions; this would result in uneven sites for anchoring the Pd precursors, and thus, the Pd NPs might partially aggregate. The lattice fringe with the d-spacing of $0.224 \mathrm{~nm}$ can be readily indexed to the (1 111 ) lattice planes of $\mathrm{Pd},{ }^{51}$ consistent with the XRD results.

Fourier transform infrared (FTIR) spectroscopy was employed to analyze the evolution of the oxygen groups on the GO surface; in the infrared spectrum of Pd/NRGO, the peaks at $1747 \mathrm{~cm}^{-1}, 1076 \mathrm{~cm}^{-1}, 841 \mathrm{~cm}^{-1}$ and $495 \mathrm{~cm}^{-1}$ are attributed to the stretching vibration of $\mathrm{C}=\mathrm{O}$, the stretching vibration of $\mathrm{C}-$ $\mathrm{O}$, the external bending vibration of $\mathrm{C}-\mathrm{H}$ and the deformation vibration of $\mathrm{C}-\mathrm{N}-\mathrm{O}$, respectively. ${ }^{52}$ There is no characteristic peak of $\mathrm{C}-\mathrm{N}-\mathrm{O}$ in the infrared spectrum of $\mathrm{Pd} / \mathrm{RGO}$. The

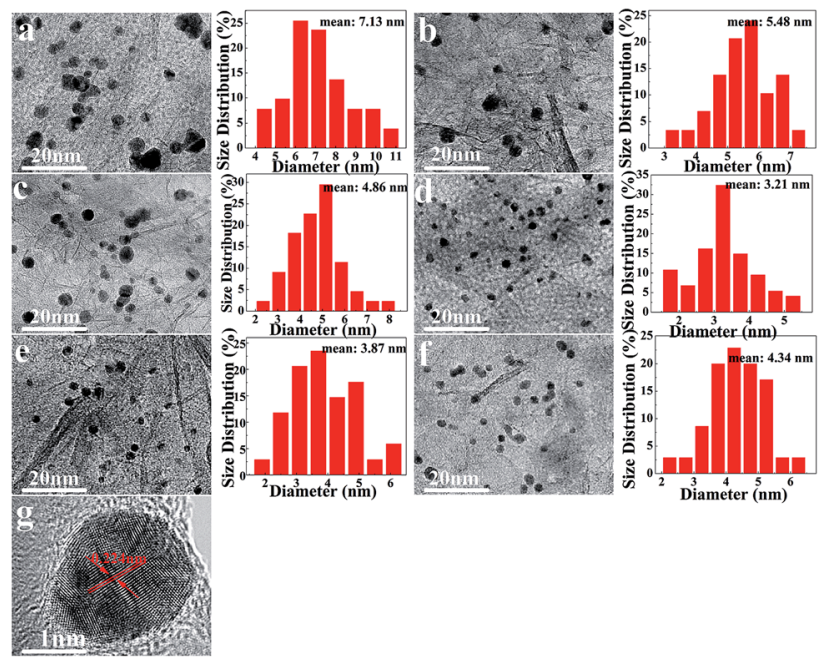

Fig. 4 TEM images and the particle size distribution of catalyst- 0 (a), catalyst-1 (b), catalyst- 2 (c), catalyst- 3 (d), catalyst -4 (e), and catalyst-5 (f); $(\mathrm{g})$ the HRTEM image of catalyst-3.

presence of the $\mathrm{C}-\mathrm{N}-\mathrm{O}$ peak confirms the incorporation of NMP into GO in the form of a covalent bond.

To investigate the composition and chemical state of especially the surface of the 3D Pd/NRGO (catalyst-3 (c)) material, XPS analysis was carried out, and the results are shown in Fig. 5d-f. To fully understand the compositions of the obtained samples, XPS measurements were employed to identify the states of $\mathrm{C}, \mathrm{N}$ and $\mathrm{O}$ in bulk materials, and the typical results are shown in Fig. $5{ }^{30}$ From the XPS survey scan results (scan not shown herein), the $\mathrm{N}$ concentration is found to be about 2 at $\%$ for Pd/NRGO, which is consistent with the elemental analysis results. The sharp peak at about $284.5 \mathrm{eV}$ in Fig. $5 \mathrm{~d}$ may be assigned to the $\mathrm{C}-\mathrm{C}$ bonds with $\mathrm{sp}^{2} / \mathrm{sp}^{3}$ hybrid carbon in the $\mathrm{C}$ 1s spectrum of 3D Pd/NRGO. The other three distinct peaks at higher binding energies can reasonably correspond to the following carbon functional groups: C-C (284.6 eV), C-N (285.9 $\mathrm{eV}){ }^{53} \mathrm{C}-\mathrm{O}(286.5 \mathrm{eV})$ and $\mathrm{O}-\mathrm{C}=\mathrm{O}(289.2 \mathrm{eV})$. This result confirms the nitrogen functionalization of graphene by NMP, and the $\mathrm{C}-\mathrm{N}$ bonds promote the dispersion of metal nanoparticles; ${ }^{32,54}$ this is mainly attributed to the pyridine-type

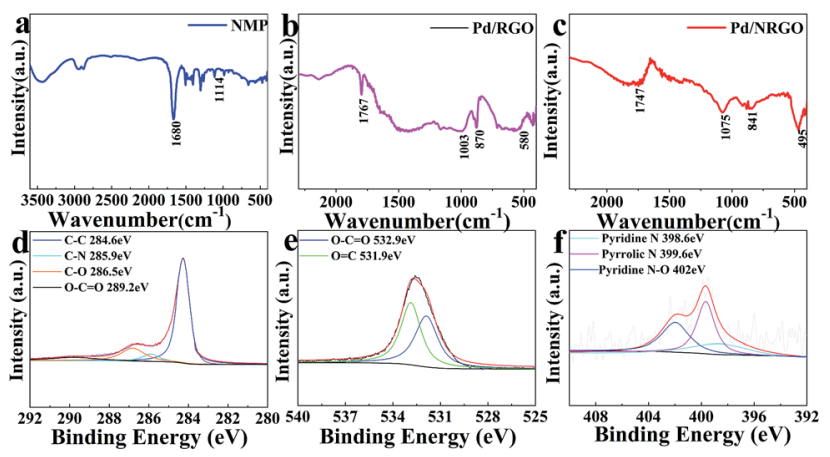

Fig. 5 FTIR spectra of NMP (a), catalyst- 0 (b) and catalyst-3 (c) and XPS core level spectra of Pd/NRGO (catalyst-3): (d) C 1s, (e) O 1s, and (f) N $1 \mathrm{~s}$. 
nitrogen species that is a good ligand and strongly interacts with metal catalysts. ${ }^{55}$ As shown in Fig. 5e, the $\mathrm{O}$ 1s spectrum can be deconvoluted into two types of oxygen: the peaks at $532.9 \mathrm{eV}$ and $531.9 \mathrm{eV}$ are associated with $\mathrm{O}-\mathrm{C}=\mathrm{O}$ and $\mathrm{O}=\mathrm{C}$, respectively. In Fig. $5 f$, the $\mathrm{N}$ 1s spectrum can be deconvoluted into three types of nitrogen: the peaks at $398.6 \mathrm{eV}, 399.6 \mathrm{eV}$ and $402 \mathrm{eV}$ are associated with pyridinic $\mathrm{N}$, pyrrolic $\mathrm{N}$ and pyridinic $\mathrm{N}-\mathrm{O}$, respectively. ${ }^{31}$ These results further demonstrate the successful grafting of NMP on the graphene sheets; this is in good agreement with the FTIR results.

The Brunauer-Emmett-Teller (BET) surface area and porous structure of the catalyst- 0 and catalyst- 3 were characterized by nitrogen adsorption-desorption analysis, and the obtained isotherms and pore distribution maps are shown in Fig. 6. As shown in Fig. 6a, the adsorption-desorption curves show that the two composites exhibit the similar type IV isotherm with an H3-type hysteresis loop in the large range of $0.2-0.95 P / P_{0}$, demonstrating the presence of mesoporous and macroporous structures in the composites. ${ }^{56}$ The BET surface areas, pore sizes and pore volumes of the catalyst- 0 and catalyst- 3 are summarized in Table 2. The test results show that the specific surface area of 3D Pd/NRGO (catalyst-3) is as high as $270.2 \mathrm{~m}^{2} \mathrm{~g}^{-1}$, whereas the specific surface area of $3 \mathrm{D} \mathrm{Pd/RGO}$ (catalyst- 0 ) is just $60.7 \mathrm{~m}^{2} \mathrm{~g}^{-1}$. Fig. 6b show the BJH fitting pore distribution map of the catalyst- 0 and catalyst-3. It can be seen from the figure that most of the pores of the catalyst- 3 have sizes in the range from $1 \mathrm{~nm}$ to $160 \mathrm{~nm}$, especially in the range from $2 \mathrm{~nm}$ to
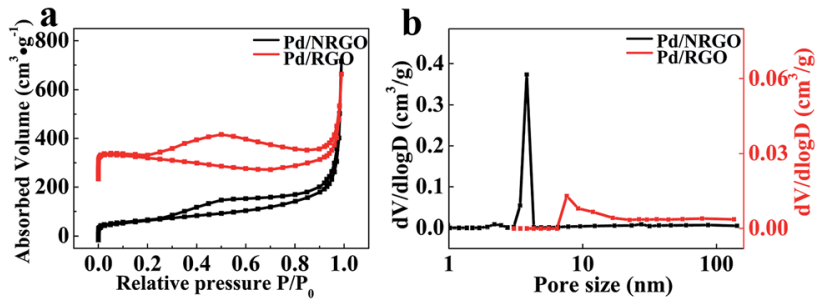

Fig. 6 (a) $N_{2}$ adsorption-desorption isotherms, inset shows the images of the catalyst-3 (A) and catalyst- 0 (B), and (b) pore size distribution of the catalyst- 0 (red line) and catalyst-3 (black line).

Table 1 The values of the $I_{D} / I_{G}$ ratio of the six catalysts with different NMP/GO mass ratios: catalyst-0 (A), catalyst-1 (B), catalyst-2 (C), catalyst-3 (D), catalyst-4 (E), and catalyst-5 (F)

\begin{tabular}{lllllll}
\hline & $\mathrm{A}$ & $\mathrm{B}$ & $\mathrm{C}$ & $\mathrm{D}$ & $\mathrm{E}$ & $\mathrm{F}$ \\
\hline $\mathrm{NMP}: \mathrm{GO}$ & $0: 1$ & $0.8: 1$ & $1.2: 1$ & $1.5: 1$ & $1.8: 1$ & $2.2: 1$ \\
$I_{\mathrm{D}} / I_{\mathrm{G}}$ & 1.42 & 1.42 & 1.45 & 1.47 & 1.49 & 1.51
\end{tabular}

Table 2 BET surface area, pore volume and average pore size of the catalyst- 0 and catalyst-3

\begin{tabular}{llll}
\hline Compound & $\begin{array}{l}\text { Surface area } \\
\left(\mathrm{m}^{2} \mathrm{~g}^{-1}\right)\end{array}$ & $\begin{array}{l}\text { Pore volume } \\
\left(\mathrm{cm}^{3} \mathrm{~g}^{-1}\right)\end{array}$ & $\begin{array}{l}\text { Average pore diameter } \\
(\mathrm{nm})\end{array}$ \\
\hline Catalyst-0 & 60.7 & 0.65 & 7.6 \\
Catalyst-3 & 270.2 & 1.13 & 3.8
\end{tabular}

$5 \mathrm{~nm}$, and the average pore diameter is $3.8 \mathrm{~nm}$. For the catalyst0 , the pore size is in the range from $2 \mathrm{~nm}$ to $160 \mathrm{~nm}$, and the average pore diameter is $7.6 \mathrm{~nm}$. The pore volumes of catalyst-3 and catalyst- 0 are $1.13 \mathrm{~cm}^{3} \mathrm{~g}^{-1}$ and $0.65 \mathrm{~cm}^{3} \mathrm{~g}^{-1}$, respectively. The cylindrical morphology of catalyst-0 (B) and catalyst- 3 (A) is shown in Fig. 6a. The volume of the nitrogen-doped graphene composite is larger than that of the undoped graphene composite. The abovementioned test results are considered as positive features in this study since the mesoporous structure with large specific surface can effectively prevent the agglomeration of Pd; this is beneficial for the exposure of more active sites for oxygen reduction and enhances the catalytic activity of oxygen reduction. This result is consistent with those of the SEM measurements.

The morphology of the catalyst- 0 and catalyst- 3 aerogel samples was investigated by SEM, and the results are displayed in Fig. 7. The SEM images reveal an interconnected porous graphene 3D framework with continuous macropores in the micrometer size range for 3D Pd/NRGO (Fig. 7a) and 3D Pd/RGO (Fig. 7b). However, close observation shows that the pores in the catalyst-3 aerogel structure are relatively uniformly dense and have a small size ranging from submicrometer to several micrometers (Fig. 7c); on the other hand, the pores in the structure of the catalyst- 0 aerogel are relatively rare and have a large pore diameter (Fig. 7d). This also confirms that the catalyst- 3 has a larger specific surface area than catalyst- 0 ; this is consistent with the results of the BET measurement. That is, $\mathrm{N}$ doped graphene can enhance the interface contact and suppress the dissolution and agglomeration of Pd NPs, ${ }^{57}$ thereby promoting the chemical activity and stability of the material.

\section{The scope of the benzyl alcohol oxidation reactions with different substituents}

The catalytic activity of 3D Pd/NRGO in the oxidation of benzyl alcohols with different substituent groups was also evaluated, and the results are shown in Table 3. As listed in Table 3, the

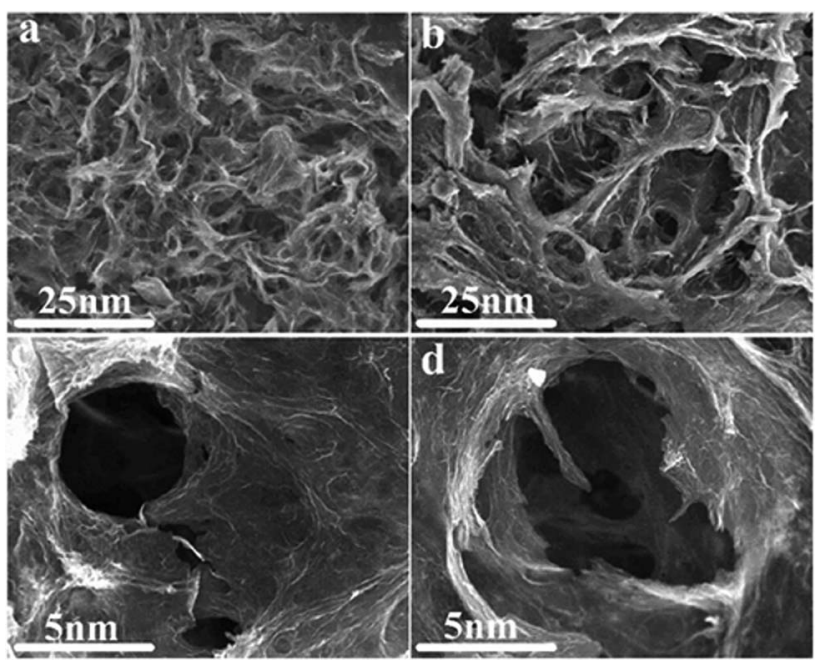

Fig. 7 The SEM images ((a) and (c)) of the catalyst- 0 and ((b) and (d)) catalyst-3. 
Table 3 Catalytic activity of Pd/NRGO in the oxidation of different alcohols

$\left.\left.\begin{array}{l}\text { Conversion } \\ (\%)\end{array}\right) \begin{array}{l}\text { Selectivity } \\ (\%)\end{array}\right)$

representative analogue of benzyl alcohol can be smoothly transformed into the corresponding aldehyde using 3D Pd/ NRGO under molecular oxygen. The catalytic activity for each case depends on the property of the substituent. ${ }^{58,59}$ The conversion of alcohol decreases according to the following order of the substituent groups: $-\mathrm{OCH}_{3}>-\mathrm{OH}>-\mathrm{CH}_{3}>-\mathrm{Cl}>$ $-\mathrm{NO}_{2}$; very few aliphatic alcohols have been studied for the oxidation reaction as they require longer time and provide lower yield as compared to aromatic alcohols (entries 6-8). ${ }^{60}$ The electron cloud density of the benzene ring is likely to increase as the electron donating ability of the substituent increases, such that the aromatic alcohol is more easily oxidized to an aromatic aldehyde. Alcohols with electron-donating substituents (entries 1-3) have higher catalytic conversion. Obviously, substituents with electron-withdrawing groups hinder the activation of hydroxyl groups in the catalytic reactions (entries 4-5). ${ }^{61,62}$

Regarding the catalytic mechanism of the Pd-catalyzed selective oxidation of benzyl alcohol, it is generally believed that the first step is the activation of alcohol on Pd and the formation of an alkoxide. ${ }^{3,65,66}$ Subsequently, the alkoxide undergoes $\beta$-elimination and is then converted to an aldehyde, which is also considered to be the rate-determining step for the overall catalytic oxidation of alcohol. Among all the substituents, the electron-donating substituents can promote the formation of a carbocation-type transition state. The effect of the substituted benzyl alcohols on the selective oxidation catalytic activity was also observed on Pd nanoparticles loaded on other materials. ${ }^{67,68}$

\section{Reusability test}

The catalytic stability test results of the prepared 3D Pd/NRGO (catalyst-3) nanocomposite are shown in the form of
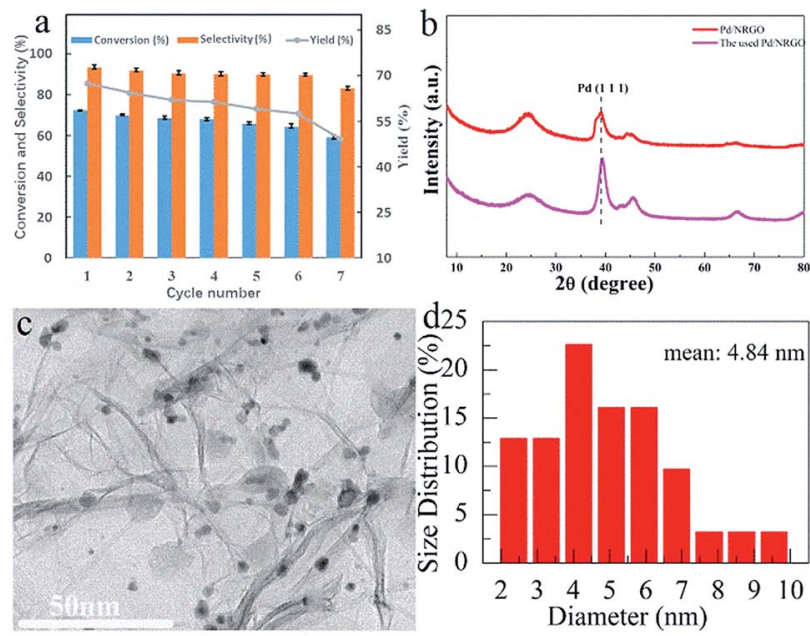

Fig. 8 (a) Reusability of catalyst-3 for the benzyl alcohol oxidation reactions, (b) XRD patterns of the fresh and used catalyst-3, (c) TEM image of catalyst- 3 after 7 cycles, and (d) the particle size distribution of catalyst-3 after 7 cycles.

a histogram ${ }^{63}$ in Fig. 8a. After seven cycles, the yield decreased to $49.3 \%$. To understand the deactivation mechanism of 3D Pd/ NRGO (catalyst-3), the XRD patterns of the fresh and used catalyst-3 (Fig. 8b) and the TEM images of 3D Pd/NRGO (catalyst-3) (Fig. 8c) were obtained after seven cycles. The XRD patterns of the fresh and used catalyst- 3 show that as compared to the fresh catalyst, the half-height width of the catalyst that has been recycled seven times is reduced, and the peak position is also shifted towards large angle. According to the DebyeScherrer formula, it can be concluded that the particle size of the catalyst after seven cycles increases to some extent; this is consistent with the results of the particle size analysis shown in the TEM image in Fig. 8c. ${ }^{64}$ The TEM image of the catalyst-3 shows that the palladium nanoparticles partially agglomerate after seven cycles, and the average particle size is $4.84 \mathrm{~nm}$. The loading of palladium in the catalyst also reduced by about 5 percent. This may be the reason for the decline in the catalyst activity.

\section{Catalytic performances of different Pd-based catalysts in the benzyl alcohol oxidation reaction}

The catalytic performances and the TOF values for the solventfree oxidation with the 3D Pd/NRGO catalyst proposed herein and other Pd-based catalysts reported in the literature are compared in Table 4. It can be seen that the 3D Pd/NRGO catalyst (Table 4, entry 7) has better catalytic performance than the other reported catalysts (Table 4, entry 5-8), with the highest turnover frequency (TOF) of $1166 \mathrm{~h}^{-1}$. However, in the literature, higher TOF values were obtained (Table 4, entry 1-4) at very high reaction temperatures. Moreover, higher reaction temperatures require the consumption of more energy. After investigating the relationship between the catalytic performance and the energy consumption, it was clear that our catalyst had better practical significance than the other catalysts reported in the literature. 
Table 4 Catalytic performances of different Pd catalysts in the benzyl alcohol oxidation reactions ${ }^{a}$

\begin{tabular}{|c|c|c|c|c|c|c|c|}
\hline Entry & Catalyst & Temp $\left({ }^{\circ} \mathrm{C}\right)$ & Time (h) & $\begin{array}{l}\text { Conversion } \\
(\%)\end{array}$ & $\begin{array}{l}\text { Selectivity } \\
(\%)\end{array}$ & TOF $\left(\mathrm{h}^{-1}\right)$ & Ref. \\
\hline 1 & Pd/SBA & 140 & 1 & 8.6 & 90.2 & 3701 & 62 \\
\hline 3 & $\mathrm{Pd} / \mathrm{Al}_{2} \mathrm{O}_{3}-\mathrm{N}$ & 120 & 8 & 80.1 & 94.3 & 1172 & 69 \\
\hline 4 & Pd/AC-ref & 110 & 6 & 48.8 & 45.9 & 1286 & 70 \\
\hline 5 & $\mathrm{Pd} / \mathrm{Fe}_{3} \mathrm{O}_{4} @ \mathrm{mCeO}_{2}$ & 90 & 7 & 80.5 & 94.8 & 554 & 59 \\
\hline 8 & Pd/RGO & 90 & 3 & 42.9 & 84.9 & 622 & This work \\
\hline
\end{tabular}

\section{Conclusions}

In summary, we have successfully synthesized threedimensional nitrogen-doped graphene as a support material to disperse $\mathrm{Pd}$ nanoparticles by a hydrothermal synthesis method with NMP as the N source and graphene oxide as the support. XPS experiments confirmed the presence of $\mathrm{N}$ elements in the framework. Raman analysis confirmed that $3 \mathrm{D}$ Pd/NRGO produced additional structure defects. TEM images show that the Pd NPs with small particle sizes are uniformly dispersed on the surface of the graphene sheets without aggregation. The SEM and BET results confirm that $3 \mathrm{D} \mathrm{Pd} /$ NRGO has more dense three-dimensional porous structures and higher surface areas. Compared to the case of the undoped 3D Pd/RGO catalyst, an enhanced catalytic activity for the benzyl alcohol oxidation reaction was obtained in the case of the 3D Pd/NRGO catalyst with an optimized composition and nanostructure. Its excellent performance is attributed to the action of nitrogen, mainly pyridinic nitrogen, which interacts strongly with metal catalysts, and the unique porous structure of the material brought by the three-dimensional structure. The 3D Pd/NRGO catalyst has the advantages of high efficiency, convenient separation and low cost and is an ideal catalyst for solving the environmental problems. In summary, the 3D Pd/ NRGO composite aerogel has significant application potential in the field of practical industrial catalysis.

\section{Conflicts of interest}

There are no conflicts to declare.

\section{Acknowledgements}

This work was financially supported by the Shanghai Natural Science Foundation (No. 18ZR1411100), Shanghai Key Laboratory of Green Chemistry and Chemical Processes and SRF for ROCS, SEM.

\section{Notes and references}

1 S. E. Davis, M. S. Ide and R. J. Davis, Green Chem., 2013, 44, 17-45.
2 J. Che, M. Hao, W. Yi, H. Kobayashi, Y. Zhou, L. Xiao and J. Fan, Chin. J. Catal., 2017, 38, 1870-1879.

3 T. Mallat and A. Baiker, Chem. Rev., 2004, 104, 3037-3058.

4 S. Hosokawa, Y. Hayashi, S. Imamura, K. Wada and M. Inoue, Catal. Lett., 2009, 129, 394-399.

5 K. Mori, T. Hara and T. Mizugaki, J. Am. Chem. Soc., 2004, 126, 10657-10666.

6 H. Wu, Q. Zhang and Y. Wang, Adv. Synth. Catal., 2005, 347, 1356-1360.

7 M. Sankar, E. Nowicka, R. Tiruvalam, Q. He, S. H. Taylor, C. J. Kiely, D. Bethell, D. W. Knight and G. J. Hutchings, Chem.-Eur. J., 2011, 17, 6524-6532.

8 Y. Huang, Q. Wei, Y. Wang and L. Dai, Carbon, 2018, 136, 150-159.

9 J. Wang, S. Kondrat, Y. Wang, G. Brett, C. Giles, J. K. Bartley, L. Lu, Q. Liu, C. J. Kiely and G. J. Hutchings, ACS Catal., 2015, 5, 3575-3587.

10 G. Wu, R. Swaidan, D. Li and N. Li, Electrochim. Acta, 2008, 53, 7622-7629.

11 D. Li, C. Yu, M. Wang, Y. Zhang and C. Pan, RSC Adv., 2014, 4, 55394-55399.

12 K. P. Loh, Q. Bao, P. K. Ang and J. Yang, J. Mater. Chem., 2010, 20, 2277-2289.

13 X. Du, C. Zhou, H. Liu, Y. Mai and G. Wang, J. Power Sources, 2013, 241, 460-466.

14 X. Wang, M. Amat, S. Wang and J. Jian, $R S C A d v .$, 2015, 5, 28247-28250.

15 D. S. Geng, Y. H. Hu, Y. L. Li, Y. Li and X. L. Sun, Electrochem. Commun., 2012, 22, 65-68.

16 K. Parvez, S. Yang, Y. Hernandez, A. Winter, A. Turchanin, X. Feng and K. Müllen, ACS Nano, 2012, 6, 9541-9550.

17 J. Xu, M. Wang, N. P. Wickramaratne, M. Jaroniec, S. Dou and L. Dai, Adv. Mater., 2015, 27, 2042-2048.

18 H. Liu, Y. Liu and D. Zhu, J. Mater. Chem., 2011, 21, 33353345.

19 C. Zhang, N. Mahmood, H. Yin, F. Liu and Y. Hou, Adv. Mater., 2013, 25, 4932-4937.

20 Y. Ma, J. R. Ran, S. Dai, M. Jaroniec and S. Z. Qiao, Angew. Chem., Int. Ed., 2015, 54, 4646-4650.

21 Z. Yang, Z. Yao, G. Li, G. Fang, H. Nie, Z. Liu, X. Zhou, X. Chen and S. Huang, ACS Nano, 2011, 6, 205. 
22 H. M. Jeong, J. W. Lee, W. H. Shin, Y. J. Choi, H. J. Shin, J. K. Kang and J. W. Choi, Nano Lett., 2011, 11, 2472-2477.

23 G. Luo, L. Liu, J. Zhang, G. Li, B. Wang and J. Zhao, ACS Appl. Mater. Interfaces, 2013, 5, 11184-11193.

24 X. Wang, L. Li and X. Zhang, Science, 2009, 324, 768-771.

25 H. Tao, C. Yan, A. W Robertson, Y. Gao, J. Ding, Y. Zhang,

T. Maa and Z. Sun, Chem. Commun., 2017, 53, 873-876.

26 L. Zhang and Z. Xia, J. Phys. Chem. C, 2011, 115, 1117011176.

27 B. Jiang, S. Song, J. Wang, Y. Xie, W. Chu, H. Li, H. Xu, C. Tian and H. Fu, Nano Res., 2014, 7, 1280-1290.

28 S. K. Movahed, M. Dabiri and A. Bazgir, Appl. Catal., A, 2014, 488, 265-274.

29 R. Nie, M. Miao, W. Du, J. Shi, Y. Liu and Z. Hou, Appl. Catal., B, 2016, 180, 607-661.

30 H. Xu, B. Yan, K. Zhan, J. Wang, S. Li, C. Wang, Y. Du, P. Yang, S. Jiang and S. Song, Appl. Surf. Sci., 2017, 416, 191-199.

31 Y. Zhao, Y. Zhou, B. Xiong, J. Wang, X. Chen, R. O'Hayre and Z. Shao, J. Solid State Electrochem., 2013, 17, 1089-1098.

32 Y. Zhou, K. Neyerlin, T. S. Olson, S. Pylypenko, J. Bult, H. N. Dinh, T. Gennett, Z. P. Shao and R. O'Hayre, Energy Environ. Sci., 2010, 3, 1437-1446.

33 Y. Xu, K. Sheng, C. Li and G. Shi, ACS Nano, 2010, 4, 43244330.

34 S. P. Sasikala, K. Huang, B. Giroire, P. Prabhakaran, L. Henry, A. Penicaud, P. Poulin and C. Aymonier, ACS Appl. Mater. Interfaces, 2016, 8, 30964-30971.

35 F. Galvanin, M. Sankar, S. Cattaneo, D. Bethell, V. Dua, G. J. Hutchingsb and A. Gavriilidis, Chem. Eng. J., 2018, 342, 196-210.

36 S. Guadix-Montero, H. Alshammari, R. Dalebout, E. Nowicka, D. J Morgan, G. Shaw, Q. He and M. Sankar, Appl. Catal., A, 2017, 546, 58-66.

$37 \mathrm{~S} . \mathrm{Wu}, \mathrm{H}$. He, X. Li, C. Yang, G. Zeng, B. Wu, S. He and L. Lu, Chem. Eng. J., 2018, 341, 126-136.

38 S. Wu, H. Li, X. Li, H. He and C. Yang, Chem. Eng. J., 2018, 353, 533-541.

39 N. I. Kovtyukhova, P. J. Ollivier, B. R. Martin, T. E. Mallouk, S. A. Chizhik, E. V. Buzaneva and A. D. Gorchinskiy, Chem. Mater., 1999, 11, 771-778.

40 H. Yang, C. Shan, F. Li, D. Han, Q. Zhang and L. Niu, Chem. Commun., 2009, 45, 3880-3882.

41 Y. Zheng, Y. Jiao, L. Ge, M. Jaroniec and S. Qiao, Angew. Chem., Int. Ed., 2013, 52, 3110-3116.

42 S. Sarkar, M. Sudolská, M. Dubecký, C. J. Reckmeier, A. L. Rogach, R. Zbořil and M. Otyepka, J. Phys. Chem. C, 2016, 120, 1303-1308.

43 M. Xiao, X. S. Du, Y. Z. Meng and K. C. Gong, New Carbon Materials, 2004, 19, 92-96.

44 M. Mirza-Aghayan, M. M. Tavana and R. Boukherroub, Catal. Commun., 2015, 69, 97-103.

45 Y. Shang, T. Li, H. Li, A. Dang, L. Zhang, Y. Yin and C. Xiong, Composites, Part B, 2016, 99, 106-111.
46 H. Zhang, J. Wang, Q. Yan, W. Zheng, C. Chen and Z. Yu, J. Mater. Chem., 2011, 21, 5392-5397.

47 K. N. Kudin, B. Ozbas, H. C. Schniepp, R. K. Prud'homme, I. A. Aksay and R. Car, Nano Lett., 2008, 8, 36-41.

48 A. Das, B. Chakraborty and A. K. Sood, Bull. Mater. Sci., 2008, 31, 579-584.

49 Z. Ni, Y. Wang, T. Yu and Z. Shen, Nano Res., 2008, 1, 273291.

50 Y. Xu, K. Sheng, C. Li and G. Shi, ACS Nano, 2010, 4, 43244330.

51 T. Teranishi and M. Miyake, Chem. Mater., 1998, 10, 599600.

52 V. H. Pham, T. V. Cuong and S. H. Hur, J. Mater. Chem., 2011, 21, 3371-3377.

53 S. Stankovich, D. A. Dikin, R. D. Piner, K. A. Kohlhaas, A. Kleinhammes, Y. Y. Jia, Y. Wu, S. T. Nguyen and R. S. Ruoff, Carbon, 2007, 45, 1558-1565.

54 B. Xiong, Y. Zhou, R. O'Hayre and Z. Shao, Appl. Surf. Sci., 2013, 266, 433-439.

55 L. Zhang, X. Liang, W. Song and Z. Wu, Phys. Chem. Chem. Phys., 2010, 12, 12055-12059.

56 H. Song, Q. Zhu, X. Zheng and X. Chen, J. Mater. Chem. A, 2015, 3, 10368-10377.

57 Z. Wu, S. Yang, Y. Sun, K. Parvez, X. Feng and M. Klaus, J. Am. Chem. Soc., 2012, 134, 9082-9085.

58 J. Xu, J. Shang, Y. Chen, Y. Wang and Y. Li, Appl. Catal., A, 2017, 542, 380-388.

59 L. Kong, C. Wang, F. Gong, W. Zhu, Y. Zhong, X. Ye and F. Li, Catal. Lett., 2016, 146, 1321-1330.

60 P. D. Lokhande, S. R. Waghmare, H. Gaikwad and P. P. Hankare, J. Korean Chem. Soc., 2012, 56, 539-541.

61 Q. Tang, T. Liu and Y. Yang, Catal. Commun., 2008, 9, 25702573.

62 Y. Chen, H. Lim, Q. Tang, Y. Gao, T. Sun, Q. Yan and Y. Yang, Appl. Catal., A, 2010, 380, 55-65.

63 Y. Lin, S. Wu, C. Yang, M. Chen and X. Li, Appl. Catal., B, 2019, 245, 71-86.

64 Y. Lin, S. Wu, X. Li, X. Wu, C. Yang, G. Zeng, Y. Peng, Q. Zhou and L. Lu, Appl. Catal., B, 2018, 227, 557-570.

65 S. Aditya, G. E. Chan-Thaw, R. Ilenia, V. Alberto and P. Laura, ChemCatChem, 2014, 6, 3464-3473.

66 D. Ferri, C. Mondelli, F. Krumeich and A. Baiker, J. Phys. Chem. B, 2006, 110, 22982-22986.

67 Q. Zhang, W. Fang, Y. Wang and H. Wan, Chin. J. Catal., 2010, 31, 1061-1070.

68 B. Karimi, H. Behzadnia, M. Bosrina and H. Vali, Chem. - Eur. J., 2012, 18, 8634-8640.

69 X. Wang, G. Wu, N. Guan and L. Li, Appl. Catal., B, 2012, 115, 7-15.

70 G. Wu, X. Wang, N. Guan and L. Li, Appl. Catal., B, 2013, 136137, 177-185.

71 Y. Hong, X. Jing, J. Huang, D. Sun, T. Odoom-Wubah, F. Yang, M. Du and Q. Li, ACS Sustainable Chem. Eng., 2014, 2, 1752-1759. 